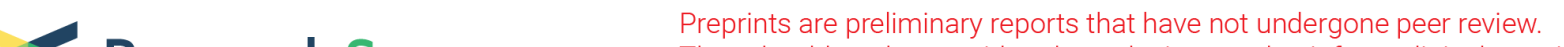 $\begin{array}{ll}\text { Research Square } & \begin{array}{l}\text { They should not be considered conclusive, used to inform clinical practice, } \\ \text { or referenced by the media as validated information. }\end{array}\end{array}$
}

\section{The prognostic significance and regulatory networks of DAP3 in hepatocellular carcinoma microenvironment based on bioinformatics analysis}

Jie Jiang

Department of Gastroenterology and Hepatology, Shanghai Tongji Hospital, Tongji University School of Medicine, Shanghai, China

\section{Bo Sun}

Department of Gastroentrology and Hepatology, Shanghai Tongji Hospital, Tongji University School of Medicine, Shanghai, China

\section{Xinyan Zhu}

Department of Gastroenterology and Hepatology, Shanghai East Hospital, Tongji University School of Medicine, Shanghai, China

\section{Yu Zhang}

Department of Gastroenterology and Hepatology, Shanghai Tongji Hospital, Tongji University School of Medicine, Shanghai, China

\section{Jie Xiong}

Department of Gastroenterology and Hepatology, Shanghai Tongji Hospital, Tongji University School of Medicine, Shanghai, China

\section{Minbo Zhang}

Gastroenterology and Hepatology, Shanghai Tongji Hospital, Tongji Uniersity School of Medicine,

Shanghai, Chiana

\section{Yiting Qian}

Department of Gastroenterology and Hepatology, Shanghai Tongji Hospital, Tongji University School of Medicine, Shanghai, China

\section{Ruilin Liu}

Department of Pulmonary, Shanghai Tongji Hospital, Tongji University School of Medicine, Shanghai, China

\section{Wenzhuo Yang ( $\triangle$ yangwenzhuo2002@163.com )}

Department of Gastroenterology and Hepatology, Shanghai Tongji Hospital, Tongji University School of Medicien, Shanghai, China https://orcid.org/0000-0001-6567-7248

\section{Research}


Keywords: DAP3, hepatocellular carcinoma, tumor microenvironment, prognosis, functional network analysis

Posted Date: September 8th, 2020

DOI: https://doi.org/10.21203/rs.3.rs-67782/v1

License: (c) (1) This work is licensed under a Creative Commons Attribution 4.0 International License. Read Full License 


\section{Abstract}

Background: Death-associated protein 3 (DAP3) is a GTP-binding protein in the component of the small subunit of the mitochondrial ribosome. Abnormal expression of DAP3 potentially correlates with carcinogenesis. However, the specific functions of DAP3 in hepatocellular carcinoma (HCC) are still not clarified.

Methods: The expression of DAP3 in different cancer types was analyzed using TIMER and Oncomine, and DAP3 expression in HCC was detected using Oncomine, GEPIA and UALCAN. DAP3 promoter methylation was shown via MEXPRESS. The effect of DAP3 on HCC prognosis was analyzed via KaplanMeier plotter, GEPIA and UALCAN. Moreover, co-expressed genes with DAP3 and their regulators, kinases, miRNA and transcription factor targets were identified using LinkedOmics. Furthermore, cBioPortal was used to detect the genetic alteration of DAP3. Finally, TIMER was utilized to evaluate the correlations between DAP3 and immune cell infiltration, while the correlation between DAP3 and three immune factors was detected through TIDISB.

Results: DAP3 expression was significantly high in several tumor tissues, including HCC. The promoter methylation level of DAP3 in HCC tissues was remarkably lower compared with normal tissues. Additionally, high DAP3 expression was related to overall survival (OS). Functional network analysis indicated that DAP3 regulated the translational elongation, ribonucleoprotein complex biogenesis, tRNA metabolic process and mitochondrial respiratory chain complex assembly. Moreover, DAP3 expression represented strong correlations with the immune cell infiltration and immune factors in HCC.

Conclusions: Our results indicated that DAP3 acted as a potential prognostic marker in HCC, laying a groundwork for further study of DAP3 in HCC.

\section{Introduction}

Liver cancer is one of the most common malignant tumors and the fourth leading cause of cancer-related deaths worldwide, threatening human health and life seriously [1, 2]. Hepatocellular carcinoma (HCC) is a common type of liver cancer, accounting for almost $90 \%[3,4]$. The major risk factors, which develop HCC, are excessive alcohol intake and virus infection, including hepatitis B or C. Although HCC patients for early detection have an access to various treatments, such as surgery, liver transplantation and radiation, the therapeutics for patients with advanced stages are not effective and the prognosis of advanced HCC patients is significantly poor, with a low 5 -year survival rate under the level of $5 \%$. Therefore, it is important to develop better biomarkers for diagnosis and prognosis of HCC.

Apoptosis is a fundamental process that plays a critical role in development and various biological functions [5]. Also, apoptosis is of great importance for the pathological conditions of many diseases, including malignancy [6]. Since then, many genes have been found and identified for their role in programmed cell death. P53, B-cell lymphoma 2 (BCL2), Bcl-2-associated X protein (BAX), and those encoding caspase $3,6,8$ and 9 are included but not limited. 
The death-associated proteins (DAPs), a novel group of proteins, have been found and they confer proapoptotic function with some common feature domains [7]. In humans, the DAPs family includes DAP1, DAP2 (DAP kinase), DAP3 and DAP5 [8]. As a member of DAPs family, DAP3 is a highly conserved protein, modulating apoptosis within the external pathway and via regulating the mitochondrial fragmentation. A study showed that DAP3, a prognosis marker of gastric cancer, is down-regulated in tumor tissue, correlated with migration and chemotherapy resistance [9]. In addition, Mokbel et al found that DAP3 acts as a tumor suppressor in breast cancer indicating that knockdown of DAP3 promotes cell invasiveness, growth, adhesion and migration and reduces the overall survival [10]. Moreover, aligned with expression and function of DAP3 in gastric cancer and breast cancer, DAP3 has similar characteristics. However, the biological function and molecular mechanisms of DAP3 in HCC are still unclear.

In the present study, we use bioinformatics approaches to explore the DAP3 expression and mutation in HCC in The Cancer Genome Atlas (TCGA) and other online databases. In addition, using multidimensional methods, we evaluate genomic alterations, function networks, and prognostic value of DAP3 in $\mathrm{HCC}$ and also explore its role in tumor immunity. Thus, our results may provide novel understanding of DAP3 in HCC, and reveal a new target for HCC diagnosis and treatment.

\section{Materials And Methods}

\section{The analysis of DAP3 expression based on Oncomine}

The mRNA and DNA copy number of DAP3 in different cancers, including HCC, were detected via Oncomine database (www.oncomine.org) [11]. We focused on several datasets, such as Guichard liver, Guichard liver 2, Roessler liver (43), Roessler liver (445) and Wurmbach liver. Differences associated with $p<0.05$ were considered significant.

\section{Survival Curve Analysis Of Dap3 Via Kaplan-meier Plotter}

Kaplan-Meier Plotter (http://kmplot.com/analysis), an online survival analysis tool, was used to assess the effects of genes on the survival rates in cancers, including $371 \mathrm{LIHC}$ [12]. The correlation between DAP3 expression and HCC patient overall survival (OS) was analyzed using Kaplan-Meier Plotter. Hazard ratios (HR) with $95 \%$ confidence intervals and log-rank $P$-value were calculated. $P$-value $<0.05$ was regarded statistically significant.

\section{The Methylation Status Of DAP3}

MEXPRESS (https://mexpress.be/) is a web tool for integrating and visualizing the expression, DNA methylation and clinical data, as well as the relationships between them [13]. In this study, MEXPRESS stool was utilized for detecting the relationship between the DAP3 expression and methylation status in HCC.

\section{Gene Correlation Identification In Gepia}


The Gene Expression Profiling Interactive Analysis (GEPIA) dataset (http://gepia.cancer-pku.cn/), an interactive web server, was used to analyze RNA sequencing expression data of 9,736 tumors and 8,587 normal samples from the TCGA and GTEx projects [14]. GEPIA was used to detect the expression of DAP3 and its effect on HCC patients OS.

\section{Validation of the expression and prognosis of DAP3 in UALCAN}

UALCAN (http://ualcan.path.uab.edu) is used to conduct in-depth analyses of gene expression data through TCGA level 3 RNA-seq and clinical data from 31 cancer types [15]. UALCAN is used to examine the relative expression and methylation of DAP3 across tumor and normal tissues, as well as in various sub-groups base on cancer race, gender, age, disease stage, tumor grade and nodal metastasis. Furthermore, UALCAN was utilized to analyze the correlation between DAP3 expression and HCC patient overall survival (OS). $P<0.05$ was regarded statistically significant.

\section{The Multi-dimensional Analysis Related To DAP3 In Linkedomics}

The LinkedOmics database (http://www.linkedomics.org/ login.php) is a Web-based platform for analyzing 32 TCGA cancer-associated multi-dimensional datasets [16]. The LinkFinder module of LinkedOmics was used to analyze the differentially expressed genes in LIHC using Pearson's correlation coefficient. And the statistical plots of individual genes were represented in volcano plots, heatmaps. The LinkInterpreter, another module of LinkedOmics that recruited Web-based Gene Set AnaLysis Toolkit, was used to analyze for GO (CC, BP and MF), KEGG pathways, kinase-target enrichment, miRNA-target enrichment and transcription factor-target enrichment via gene set enrichment analysis (GSEA). The rank criterion was a $P<0.05$, and 500 stimulations were conducted.

\section{Genomic Alterations Of DAP3 In Cbioportal}

The cBio Cancer Genomics Portal (cBioPortal) (http://cbioportal.org) is an open-access resource for exploring the multidimensional cancer genomics data sets [17]. The alterations of DAP3, including mutation, copy number variation (CNV) and mRNA expression, in TCGA LIHC were analyzed using cBioPortal. The tab OncoPrint shows an overview of genetic alterations per sample in DAP3.

\section{Integration Of Protein-protein Interaction Network}

The protein-protein interaction network of DAP3 was analyzed using String and GeneMANIA. String (https://string-db.org/cgi/input.pl) is a dataset to predict PPI including physical and functional associations, deriving from computational predictions, knowledge transfer between organisms, and interactions aggregated from other (primary) databases [18]. String was used to perform a PPI network between DAP3 and other important proteins. In addition, the protein-protein interaction (PPI) network to predict the gene functions was constructed by GeneMANIA (http://www.genemania.org) [19]. his stool was used to evaluate DAP3 function via the gene networks based on physical interactions, co-expression, predictions, colocalization, and genetic interaction. 


\section{The Immune Analysis Related To The DAP3 Expression}

The TIMER dataset (www.cistrome.shinyapps.io/timer/) is a web server to explore the tumor-infiltrating immune cells (TILCs) based on gene expression profiles across 32 cancer types [20]. The mRNA of DAP3 in different cancers, including HCC, was analyzed via TMIER database. The TIMER database was used to detect the relation between DAP3 expression and the infiltration of immune cells, including $\mathrm{CD} 4^{+} \mathrm{T}$ cells, $\mathrm{CD}^{+} \mathrm{T}$ cells, B cells, neutrophils, macrophages, and dendritic cells. Furthermore, the "SCNA" module of TIMER database was used to measure the correlation between the infiltration levels of immune cells and the mRNA level of DAP3. Furthermore, the correlation between DAP3 expression and immunomodulators and chemokines was analyzed using TISIDB database (http://cis.hku.hk/TISIDB/index.php), which is a web portal integrating genes of tumor and immune system interactions from seven public databases [21].

\section{Results}

\section{The expression of DAP3 in HCC and various cancers}

We used TIMER database to detect the mRNA expression of DAP3 in various cancers. The results showed that DAP3 mRNA levels were significantly higher in BLCA (Bladder urothelial carcinoma), BRCA (Breast invasive carcinoma), $\mathrm{CHOL}$ (Cholangiocarcinoma), COAD (Colon adenocarcinoma), ESCA (Esophageal carcinoma), HNSC (Head and Neck squamous cell carcinoma), KICH (Kidney Chromophobe), KIRC (Kidney Chromophobe), LIHC (Liver hepatocellular carcinoma), LUAD (Lung adenocarcinoma), LUSC (Lung adenocarcinoma), PRAD (Prostate adenocarcinoma), READ (Rectum adenocarcinoma), SKCM (Skin Cutaneous Melanoma), STAD (Stomach adenocarcinoma), THCA (Thyroid carcinoma), UCEC (Uterine Corpus Endometrial Carcinoma) (Fig. 1A). The analysis using Oncomine database also demonstrated the abnormal mRNA level of DAP3 in various cancers (Fig. 1B). Results mentioned above represented the dysregulation of DAP3 in various cancer tissues.

We detected the expression of DAP3 in normal and tumor tissues of HCC from TCGA and GTEx databases using GEPIA. The result showed that DAP3 mRNA expression in liver tissues was higher than that in normal tissues (Fig. 2A). Moreover, we found that DAP3 expression in HCC tumor tissues was higher than that in adjacent normal tissues by using Oncomine (Supplement Fig. 1). Aligned with the results in GEPIA and Oncomine, data from UALCAN indicated that the mRNA expression of DAP3 in cancer tissues was also obviously up-regulated compared to that in normal tissues. Furthermore, we conducted a subgroup analysis of multiple clinic-pathological features in HCC samples from UALCAN (Fig. 2B). The results of subgroup analysis based on race, gender, age, disease stage, tumor grade and nodal metastasis indicated that the DAP3 mRNA levels were remarkably higher in HCC patients than those in normal controls (Fig. 2C-H). Taken together, DAP3 may act as a potential biomarker to diagnose HCC.

To better understand the role of DAP3 in HCC, we detected its copy-number variation (CNV) and methylation levels. We found that DNA copy number variation (CNV) of DAP3 were significantly higher in 
HCC tissues than in normal tissues ( $p \otimes 0.01)$ (Fig. 3A). In addition, the result revealed that the methylation level of DAP3 promoter regions was modified (Fig. 3B). Interestingly, the DNA methyltransferase (DNMT) expression was positively related with DAP3 in HCC (Fig. 3C).

\section{Prognostic Value Of DAP3 Expression In HCC Patients}

To identify the prognostic significance of DAP3 in HCC patients, we analyzed survival curves for DAP3 expression and survival using three public databases, including the Kaplan-Meier Plotter database, the GEPIA database, and UALCAN database. Results from the databases revealed that patients with high DAP3 expression had a shorter overall survival (OS) than those with low DAP3 expression, indicating that high expression of DAP3 was associated with poor prognosis (Fig. 4). Thus, DAP3 expression may be recognized as a potential prognostic indicator in HCC.

\section{GO and KEGG pathway analyses of co-expression genes correlated with DAP3 in HCC}

The function module of LinkedOmics was used to explore the biological meaning of DAP3 correlated with other genes in HCC. As shown in the volcano plot, the expression of 2130 genes (dark red dots) was positively correlated with that of $D A P 3$, whereas the expression of 4027 genes (dark green dots) was negatively correlated (false discovery rate, FDR < 0.05) (Fig. 5A). The top 50 significant genes positively and negatively correlated with $D A P 3$ were displayed in the heat map (Fig. 5B, C). These results implied that DAP3 played a critical effect on the transcriptome (Supplementary Table 1). DAP3 expression showed a strong positive association with expression of FLAD1 $(\mathrm{r}=0.7095, p=1.579 \mathrm{E}-56)$, C1 orf $43(\mathrm{r}=$ 0.6883, $p=5.634 \mathrm{E}-51)$, and $\operatorname{HAX} 1(\mathrm{r}=0.6773, p=8.596 \mathrm{E}-50)$, and the results revealed the negative correlation between DAP3 expression and DCUN1D3 $(r=-0.4614, p=1.963 \mathrm{E}-20)$, DPF3 $(r=-0.442, p=$ 1.072E-18), and $G A B 1$ ( $r=-0.4235, p=3.797 \mathrm{E}-17$ ) (Fig. 6), reflecting changes in adhesion, proliferation, apoptosis, and cell cycle. In addition, the expression of top 3 positive genes also affected the overall survival on GEPIA database (Supplement Fig. 2). Furthermore, GO term analysis of GSEA indicated that genes differentially expressed correlated with $D A P 3$ were located prominently in translational elongation, ribonucleoprotein complex biogenesis, tRNA metabolic process and mitochondrial respiratory chain complex assembly (Fig. 7A-C and Supplementary Table 2-4). Moreover, KEGG pathway results displayed enrichment in ribosome, proteasome, aminoacyl-tRNA biosynthesis, spliceosome, etc (Fig. 7D and Supplementary Table 5).

\section{Regulators of kinases, miRNAs, transcription factors associated with DAP3 in HCC}

In order to explore the targets of DAP3 in HCC, we analyzed the possible kinases, miRNAs, and transcription factors of $D A P 3$ using LinkedOmics. The top 5 kinases such as 3-phosphoinositide dependent protein kinase 1 (PDPK1), mitogen-activated protein kinase 3 (MAPK3), ribosomal protein S6 kinase A1 (RPS6KA1), protein tyrosine kinase 6 (PTK6) and LYN proto-oncogene, Src family tyrosine kinase (LYN1), were enriched by GSEA for DAP3 co-expressed genes (Table 1 and Supplementary Table 6). And the top 5 enrichments of miRNA were associated with (TGTTTAC) MIR-30A-5P, MIR-30C, MIR-30D, MIR-30B, MIR-30E-5P, (TACTTGA) MIR-26A, MIR-26B, (ACAACTT) MIR-382, (GCACTTT) MIR-175P, MIR-20A, MIR-106A, MIR-106B, MIR-20B, MIR-519D, and (ATGTTTC) MIR-494 (Table 1 and 
Supplementary Table 7). Moreover, the top 5 correlated transcript factor-target networks were GGAANCGGAANY_UNKNOWN, V\$SOX5_01, V\$P53_DECAMER_Q2, V\$HNF3_Q6, and V\$DBP_Q6 (Table 1 and Supplementary Table 8). One recent study showed that transcript factor OCT1 was associated with the development and progression of HCC. Thus, DAP3 may play an important role in hepatocarcinogenesis via these factors.

Table 1

The Kinase, miRNA and transcription factor-target networks of DAP3 in hepatocellular carcinoma (LinkedOmics).

\begin{tabular}{|c|c|c|c|}
\hline Enriched Category & Geneset & LeadingEdgeNum & $\begin{array}{l}P \\
\text { value }\end{array}$ \\
\hline \multirow[t]{5}{*}{ Kinase Target } & Kinase_PDPK1 & 7 & 0.0077 \\
\hline & Kinase_MAPK3 & 50 & 0 \\
\hline & Kinase_RPS6KA1 & 17 & 0.0087 \\
\hline & Kinase_PTK6 & 3 & 0.0037 \\
\hline & Kinase_LYN & 24 & 0 \\
\hline \multirow[t]{5}{*}{ MiRNA Target } & $\begin{array}{l}\text { TGTTTAC,MIR-30A-5P,MIR-30C,MIR-30D,MIR- } \\
\text { 30B,MIR-30E-5P }\end{array}$ & 193 & 0 \\
\hline & TACTTGA,MIR-26A,MIR-26B & 110 & 0 \\
\hline & ACAACTT,MIR-382 & 24 & 0 \\
\hline & $\begin{array}{l}\text { GCACTTT,MIR-17-5P,MIR-20A,MIR-106A,MIR- } \\
\text { 106B,MIR-20B,MIR-519D }\end{array}$ & 234 & 0 \\
\hline & ATGTTTC,MIR-494 & 46 & 0 \\
\hline \multirow{5}{*}{$\begin{array}{l}\text { Transcription } \\
\text { Factor Target }\end{array}$} & GGAANCGGAANY_UNKNOWN & 34 & 0 \\
\hline & V\$SOX5_01 & 109 & 0 \\
\hline & V\$P53_DECAMER_Q2 & 80 & 0 \\
\hline & V\$HNF3_Q6 & 82 & 0 \\
\hline & V\$\$DBP_Q6 & 92 & 0 \\
\hline
\end{tabular}

\section{Genomic Alterations Of DAP3 In HCC}

cBioPortal was used to analyze the alternation of DAP3. DAP3 was altered in 151 of 348 (43\%) HCC patients and the parameters of DAP3 alternation contained mutation, copy-number variations, and mRNA expression (Fig. 8A). As shown in Fig. 8B, the expression of DAP3 in gain or amplification group was higher than that in the diploid group. Furthermore, we examined the frequency distribution of DAP3 CNV 
patients in different grade and stage groups, and found that $D A P 3 \mathrm{CNV}$ alteration was closely related with the high occurrence and an early-event (Fig. 8C).

\section{Networks Analysis Of DAP3 With Interacted Genes}

A PPI network was conducted using the STRING to identify the interactions between DAP3 and 20 coexpressed genes in HCC. The results showed that DAP3, interacting with mitochondrial ribosomal genes, was mainly involved in mitochondrial ribosomal functions (Fig. 9A). In addition, the PPI network analysis by GeneMANIA revealed that DAP3 was also associated with apoptosis, mitochondrial ribosomal functions, assembly of the proteasome complex, as well as arachidonic acid pathway (Fig. 9B).

\section{DAP3 expression associated with the infiltration of immune cell in HCC}

There were growing studies showing that the immune system played an important role in the tumor development and progression, and the survival times. Therefore, we performed a comprehensive analysis to investigate the relationship between DAP3 and immune cells using TIMER database. We selected six immune cells, which infiltrates tumors frequently, including $C D 4^{\square} T$ cell, $C D 8^{\square} T$ cell, neutrophil, macrophage, B cell and Dendritic cell. The results from TIMER database showed that DAP3 expression was not correlated with tumor purity (Cor $=0.045, p=4.02 \mathrm{E}-01$ ) and $\mathrm{CD} 8^{\square} \mathrm{T}$ cell (Cor $=0.009, p=8.70 \mathrm{E}-01$ ) in HCC. In addition, DAP3 expression was positively correlated with the infiltration of CD4 ${ }^{\square} \mathrm{T}$ cell (Cor = $0.202, p=1.57 \mathrm{E}-04$ ), neutrophil (Cor $=0.198, p=2.12 \mathrm{E}-04$ ), macrophage (Cor $=0.223, p=3.34 \mathrm{E}-05)$, $\mathrm{B}$ cell (Cor $=0.158, p=3.26 \mathrm{E}-03$ ) and Dendritic cell (Cor $=0.126, p=1.98 \mathrm{E}-02)$ (Fig. 10A). These results represented that DAP3 expression significantly correlated with the levels of the dominant immune cells infiltration. Furthermore, we examined relationship of the somatic copy number alterations (SCNA) in DAP3 with tumor infiltration levels. Particularly, DAP3 SCNA was remarkably correlated with the infiltrating levels of $\mathrm{CD} 8^{\square} \mathrm{T}$ cell, neutrophil, macrophage and Dendritic cell (Fig. 10B). Additionally, the top 3 positive or negative genes of DAP3 represented the significant correlations with tumor purity and varying degree with immune cells (Supplementary Fig. 3A). Furthermore, CNV of top three positive genes showed significant correlations with infiltrating levels of immune cells (Supplementary Fig. 3B).

\section{Correlations Between DAP3 Expression And Immune Factors}

Previous studies had shown that chemokines could affect various tumor biology by interacting with receptors and recruiting immune cells. Aforementioned results showed that DAP3 expression was correlated with immune cells, we then examined the relationship between DAP3 expression and chemokines using TISIDB database. As shown in heatmap of chemokines and various cancers, we focused the relationship between chemokines and DAP3 (Fig. 11A). Then, we analyzed the correlation between them after filtering for $\mid \pm$ rho $\mid \geq 0.1$ and $p<0.05$. We found that DAP3 was positively associated with CCL15, CCL16, CXCL16 and CXCL17, and DAP3 was negatively associated with CCL2, CCL4, CCL5, CCL13, CCL18, CCL22, CCL23, CXCL1, CXCL6, CXCL10, CXCL12, CXCL14, XCL2 (Fig. 11A). These data suggested that DAP3 may modulate tumor biology by dysregulating chemokines. 
In addition to chemokines, immune checkpoints also modulated tumor microenvironment and promoted the evasion of tumor cells from immune surveillance. Therefore, we detected the relationship among DAP3 and immune checkpoints including immunoinhibitors and immunostimulants using TISIDB database. The heatmap of DAP3 and immune checkpoint genes in various cancers containing HCC were as shown in Fig. 11B and C. Surprisingly, DAP3 was significantly correlated with immune checkpoint genes including immunoinhibitory genes (Fig. 11B) and immunostimulatory genes (Fig. 11C). These results revealed that DAP3 correlated with chemokines and immune checkpoints played a critical role in the development, progression and survival of HCC patients.

\section{Discussion}

As one of the most common form of cancers, HCC threatens human health and the underlying mechanisms are still unknown due to the complex of its molecular and cellular heterogeneity. The apoptosis genes have been reported to take an important role in oncogenesis in many cancers [22]. DAP3, a member of the death-associated protein (DAP) family, represents a pro-apoptotic function, which indicates that this gene is expected as a potential target for cancer research and treatment. However, the role of DAP3 in HCC has not been studied. Therefore, we performed bioinformatics analysis to investigate the functions of DAP3 in HCC and its regulatory network to direct future research and improve prognostic accuracy in $\mathrm{HCC}$ using public sequencing data.

It is difficult to diagnose HCC at early stage for a lack of markers. Though alpha-fetoprotein (AFP) has long been regarded as an indicator for early screening of HCC, almost $30 \%$ of HCC patients are AFPnegative [23]. Therefore, new biomarkers for early diagnosis of HCC are needed. In the present study, we conduct bioinformatic analyses to explore the expression of DAP3 in HCC patients and results show that the mRNA level of DAP3 in HCC tissues is remarkably increased compared to that in normal tissues. In addition, DAP3 transcription level is strongly correlated with clinic pathological features of HCC, including race, gender, age, disease stage, tumor grade and nodal metastasis. The alteration of the number of copies in genomic regions may influence the tumor biological behaviors such as cell growth, proliferation, metastasis, and recurrence [24]. Copy-number variation (CNV) of DAP3 from Guichard liver, Guichard liver 2 and TCGA liver was evaluated to better understand the role of DAP3 in liver carcinogenesis. DAP3 CNVs in $\mathrm{HCC}$ are significantly higher than those in normal liver tissue. Moreover, DNA methylation modification in the genes is another aspect affecting tumor development and progression. Yu et al found that EZH1 regulated leukemia by methylating the lysine 43 of AML1-ETO [25]. We find that the promoter methylation levels of DAP3 in HCC tissues are lower than that in normal liver tissues and DAP3 expression is positively related with DNMT expression. Additionally, high DAP3 expression is related to poor survival in HCC patients in Kaplan-Meier, GEPIA and UALCAN database. Above the mentioned results, we speculate that DAP3 may act as a potentially diagnostic marker of HCC based on the mRNA levels, CNVs and promoter methylation levels, and a prognostic marker.

Both GO and KEGG pathway enrichment analysis are used to detect functions of DAP3 in HCC. The results show that the functional network of DAP3 participate primarily in the translational elongation, 
ribonucleoprotein complex biogenesis, tRNA metabolic process and mitochondrial respiratory chain complex assembly. As we have known, DAP3, a highly conserved protein, regulates the mitochondrial fragmentation. It was reported that DAP3, localized in the mitochondrial, modulated apoptosis by affecting mitochondrial-encoded protein synthesis and mitochondrial dynamics [26].

Cancer cells have features of genomic instability and mutagenesis, and transcription factors can help stabilize and repair genomic DNA, and inhibit cell cycle progression [27, 28]. Thus, we seek to characterize the kinase targets of DAP3 in HCC and find that DAP3 in HCC is associated with a network of kinases including PDPK1, MAPK3, RPS6KA1, PTK6, and LYN1. Previous studies have provided data about the relation between cancers and these kinases [29-31]. In fact, Rosenberg study demonstrates a key role of the kinase MAPK3 in mediating the development and progression of breast cancer via genomic stability and repair [32]. Also, the up-regulation of MAPK3 contributes to CDDP resistance in gastric cancer [33]. In HCC, DAP3 may regulate DAN replication, repair, genomic stability, cell cycle progression and the epithelial-mesenchymal transition via these kinases.

Next, we seek to characterize the transcription factors targets of DAP3 in HCC and find that P53 is one of the most important transcription factors, which reveals a key role in the DAP3 network regulation, which reveals a key role in the DAP3 network regulation. The expression of cell cycle regulatory factors in tumor cells is abnormal, contributing to cell cycle disorder, which affects the tumor biological behaviors, including proliferation, differentiation and apoptosis. P53 acts as a critical role in the cell cycle regulation network [34]. The occurrence and development of HCC are affected by the abnormal expression of P53 [35]. In addition, P53 is a prognostic marker of HCC [36]. Therefore, our results indicate that DAP3 may act through these transcription factor targets to affect the development and progression of HCC.

Except transcription factor targets, miRNAs also play an important role in human carcinogenesis via posttranscriptional regulation. Various studies have confirmed that miRNAs are involved in tumor proliferation, apoptosis, metastasis, invasion and et al [37-39]. Liu study shows that miR-363 may inhibit HCC migration and invasion through E2F Transcription Factor 3[40]. Our results show that these miRNAs participate in the development and progression of HCC via proliferation, migration, apoptosis, metastasis, invasion [41-45]. Therefore, we speculate that DAP3 may affect HCC via the changes of miRNAs.

Increasing evidence suggests that tumor microenvironment is greatly important in cancers and it is significantly affected by immune alterations[46]. Studies show that immune cell infiltration is remarkably responsible for immunotherapy and clinical outcome $[47,48]$. Therefore, we analyze the DAP3 expression correlated with the infiltration status of immune cells in HCC and the results reveal that the expression of DAP3 is positively related with the infiltration of the six immune cell types, including B cells, CD ${ }^{+} \mathrm{T}$ cells, $\mathrm{CD}^{+}{ }^{+} \mathrm{T}$ cells, macrophages, neutrophils, and dendritic cells. Luster's study shows that chemokines can mediate immune cells migration and localization [49]. Then we identify the association between DAP3 and chemokines in HCC and find that DAP3 expression is correlated with many chemokines. These results indicate that DAP3 may influence the immune response via regulating immune cell infiltration and the expression of chemokines. 
Tumor microenvironment is affected by not only chemokines but also immunological checkpoints. The expression of inhibitory immune checkpoint proteins in tumor tissue is different from that in normal tissue, showing that it is changed in tumor microenvironment [50]. Cancers evade immune response and contribute to the progression of cancer through the overexpression of inhibitory ligands [51, 52].

Therefore, we analyze the correlation among the immunological checkpoints and DAP3 expression in $\mathrm{HCC}$. The results represent that DAP3 expression is significantly correlated with many immune checkpoint proteins, indicating that DAP3 may affect HCC biological function via the immune escape.

There are some limitations to our study. Firstly, due to the small sample sizes of tumors, large sample sizes are needed in public databases. Secondly, the role of DAP3 in HCC lacks the tests of clinical samples. Thirdly, we do not confirm the role of DAP3 in HCC in vitro and in vivo.

\section{Conclusions}

In conclusion, the results indicated that DAP3 expression is increased in HCC and DAP3 may act as an independent prognostic biomarker in HCC. Moreover, DAP3 may affect the development and progression of HCC via various kinases, miRNAs and transcript factors. Additionally, DAP3 plays an important role in the tumor microenvironment by the infiltration of immune cells, chemokines, and immune checkpoints. To our knowledge, we first find that DAP3 functions as a modulator in HCC. Therefore, targeting DAP3 may have therapeutic promise for HCC.

\section{Abbreviations}

Death-associated protein 3: DAP3; hepatocellular carcinoma: HCC; overall survival: OS; B-cell lymphoma 2: BCL2; Bcl-2-associated X protein: BAX; death-associated proteins: DAPs; The Cancer Genome Atlas: TCGA; Hazard ratios: HR; Gene Expression Profiling Interactive Analysis: GEPIA; cBio Cancer Genomics Portal: cBioPortal; copy number variation: CNV; tumor-infiltrating immune cells: TILCs; Bladder urothelial carcinoma: BLCA; Breast invasive carcinoma: BRCA; Cholangiocarcinoma: $\mathrm{CHOL}$; Colon adenocarcinoma: COAD; Esophageal carcinoma: ESCA; Head and Neck squamous cell carcinoma: HNSC; Kidney Chromophobe: KICH; Kidney Chromophobe: KIRC; Liver hepatocellular carcinoma: LIHC; Lung adenocarcinoma: LUAD; Lung adenocarcinoma: LUSC; Prostate adenocarcinoma: PRAD; Rectum adenocarcinoma: READ; Skin Cutaneous Melanoma: SKCM; Stomach adenocarcinoma: STAD; Thyroid carcinoma: THCA; Uterine Corpus Endometrial Carcinoma: UCEC; DNA methyltransferase: DNMT; SrC family tyrosine kinase: LYN1; 3-phosphoinositide dependent protein kinase 1: PDPK1; mitogen-activated protein kinase 3 : MAPK3; ribosomal protein S6 kinase A1: RPS6KA1; protein tyrosine kinase 6: PTK6.

\section{Declarations}

\section{Acknowledgments}

Not applicable. 


\section{Authors' Contributions}

Jie Jiang collected, analyzed and discussed the data, and wrote and reviewed the manuscript; Xinyan Zhu wrote and reviewed the manuscript; Jie Xiong wrote and reviewed the manuscript; Bo Sun, Yu Zhang, Minbo Zhang, and Yiting Qian collected, analyzed and discussed the data, and reviewed the manuscript. Wenzhuo Yang and Ruilin Liu conceived, designed this study, and wrote and reviewed the manuscript. All authors read and approved the final manuscript.

\section{Funding}

This work was supported by the National Natural Science Foundation of China (grant numbers: \#81873567 to Wenzhuo Yang, \#81700509 to Jie Xiong) and Municipal Health Commission of Shanghai (\#201640117 to Wenzhuo Yang)

\section{Availability of data and materials}

We appreciated the authors, who provided these data series, and the authors of the databases used in this article. They completed this research with great efforts.

\section{Ethics approval and consent to participate}

Not applicable.

\section{Consent for publication}

Not applicable.

\section{Competing interests}

The authors declare that they have no competing interests.

\section{Author details}

${ }^{1}$ Department of Gastroenterology and Hepatology, Shanghai Tongji Hospital, Tongji University School of Medicine, Shanghai 200065, China.

2 Department of Pulmonary, Shanghai Tongji Hospital, Tongji University School of Medicine, Shanghai 200065, China.

${ }^{3}$ Department of Gastroenterology and Hepatology, Shanghai East Hospital, Tongji University School of Medicine, Shanghai 200065, China.

\section{References}


1. Llovet JM, Montal R, Sia D, Finn RS. Molecular therapies and precision medicine for hepatocellular carcinoma. Nature Reviews Clinical Oncology. 2018; 15:599-616.

2. Bray F, Ferlay J, Soerjomataram I, Siegel RL, Torre LA, Jemal A. Global cancer statistics 2018 : GLOBOCAN estimates of incidence and mortality worldwide for 36 cancers in 185 countries. CA: a cancer journal for clinicians. 2018; 68:394-424.

3. Wang J-h, Zhong X-p, Zhang Y-f, Wu X-I, Li S-h, Jian P-e, Ling Y-h, Shi M, Chen M-s, Wei W. Cezanne predicts progression and adjuvant TACE response in hepatocellular carcinoma. Cell death \& disease. 2017; 8:e3043-e3043.

4. Llovet J, Zucman-Rossi J, Pikarsky E, Sangro B, Schwartz M, Sherman M, Gores G: Hepatocellular carcinoma. Nature reviews Disease primers. 2016; 2: 16018.

5. Morris AB, Farley CR, Pinelli DF, Adams LE, Cragg MS, Boss JM, Scharer CD, Fribourg M, Cravedi P, Heeger PS. Signaling through the Inhibitory Fc Receptor FcyRIIB Induces CD8+ T Cell Apoptosis to Limit T Cell Immunity. Immunity. 2020; 52:136-150. e136.

6. Liang L, He Y, Wang H, Zhou H, Xiao L, Ye M, Kuang Y, Luo S, Zuo Y, Feng P. The Wee1 kinase inhibitor MK1775 suppresses cell growth, attenuates stemness and synergises with bortezomib in multiple myeloma. British Journal of Haematology. 2020;

7. Woo Lee J, Hwa Soung Y, Young Kim S, Woo Nam S, Sang Park W, Young Lee J, Jin Yoo N, Hyung Lee S. Mutational analysis of proapoptotic death associated protein 3 (DAP3) P-loop domain in common human carcinomas. Acta Oncologica. 2006; 45:489-490.

8. Levy-Strumpf N, Kimchi A. Death associated proteins (DAPs): from gene identification to the analysis of their apoptotic and tumor suppressive functions. Oncogene. 1998; 17:3331-3340.

9. Jia Y, Ye L, Ji K, Zhang L, Hargest R, Ji J, Jiang WG. Death-associated protein-3, DAP-3, correlates with preoperative chemotherapy effectiveness and prognosis of gastric cancer patients following perioperative chemotherapy and radical gastrectomy. British journal of cancer. 2014; 110:421-429.

10. Wazir U, Sanders AJ, Wazir A, Ye L, Jiang WG, Ster IC, Sharma AK, Mokbel K. Effects of the knockdown of death-associated protein 3 expression on cell adhesion, growth and migration in breast cancer cells. Oncology reports. 2015; 33:2575-2582.

11. Rhodes DR, Kalyana-Sundaram S, Mahavisno V, Varambally R, Yu J, Briggs BB, Barrette TR, Anstet MJ, Kincead-Beal C, Kulkarni P. Oncomine 3.0: genes, pathways, and networks in a collection of 18,000 cancer gene expression profiles. Neoplasia (New York, NY). 2007; 9:166.

12. Lánczky A, Nagy Á, Bottai G, Munkácsy G, Szabó A, Santarpia L, Győrffy B. miRpower: a web-tool to validate survival-associated miRNAs utilizing expression data from 2178 breast cancer patients. Breast cancer research and treatment. 2016; 160:439-446.

13. Koch A, Jeschke J, Van Criekinge W, van Engeland M, De Meyer T. MEXPRESS update 2019. Nucleic acids research. 2019; 47:W561-W565.

14. Tang Z, Li C, Kang B, Gao G, Li C, Zhang Z. GEPIA: a web server for cancer and normal gene expression profiling and interactive analyses. Nucleic acids research. 2017; 45:W98-W102. 
15. Chandrashekar DS, Bashel B, Balasubramanya SAH, Creighton CJ, Ponce-Rodriguez I, Chakravarthi BV, Varambally S. UALCAN: a portal for facilitating tumor subgroup gene expression and survival analyses. Neoplasia. 2017; 19:649-658.

16. Vasaikar SV, Straub P, Wang J, Zhang B. LinkedOmics: analyzing multi-omics data within and across 32 cancer types. Nucleic acids research. 2018; 46:D956-D963.

17. Gao J, Aksoy BA, Dogrusoz U, Dresdner G, Gross B, Sumer SO, Sun Y, Jacobsen A, Sinha R, Larsson E. Integrative analysis of complex cancer genomics and clinical profiles using the cBioPortal. Sci Signal. 2013; 6:pl1-pl1.

18. Szklarczyk D, Gable AL, Lyon D, Junge A, Wyder S, Huerta-Cepas J, Simonovic M, Doncheva NT, Morris JH, Bork P. STRING v11: protein-protein association networks with increased coverage, supporting functional discovery in genome-wide experimental datasets. Nucleic acids research. 2019; 47:D607-D613.

19. Warde-Farley D, Donaldson SL, Comes O, Zuberi K, Badrawi R, Chao P, Franz M, Grouios C, Kazi F, Lopes CT. The GeneMANIA prediction server: biological network integration for gene prioritization and predicting gene function. Nucleic acids research. 2010; 38:W214-W220.

20. Li T, Fan J, Wang B, Traugh N, Chen Q, Liu JS, Li B, Liu XS. TIMER: a web server for comprehensive analysis of tumor-infiltrating immune cells. Cancer research. 2017; 77:e108-e110.

21. Ru B, Wong CN, Tong Y, Zhong JY, Zhong SSW, Wu WC, Chu KC, Wong CY, Lau CY, Chen I. TISIDB: an integrated repository portal for tumor-immune system interactions. Bioinformatics. 2019; 35:42004202.

22. Ashkenazi A, Fairbrother WJ, Leverson JD, Souers AJ. From basic apoptosis discoveries to advanced selective BCL-2 family inhibitors. Nature Reviews Drug Discovery. 2017; 16:273.

23. Luo P, Yin P, Hua R, Tan Y, Li Z, Qiu G, Yin Z, Xie X, Wang X, Chen W. A Large-scale, multicenter serum metabolite biomarker identification study for the early detection of hepatocellular carcinoma. Hepatology. 2018; 67:662-675.

24. Hu L, Yao X, Huang H, Guo Z, Cheng X, Xu Y, Shen Y, Xu B, Li D. Clinical significance of germline copy number variation in susceptibility of human diseases. Journal of Genetics and Genomics. 2018; 45:3-12.

25. Dou L, Yan F, Pang J, Zheng D, Li D, Gao L, Wang L, Xu Y, Shi J, Wang Q. Protein lysine 43 methylation by EZH1 promotes AML1-ETO transcriptional repression in leukemia. Nature communications. 2019; 10:1-15.

26. Xiao L, Xian H, Lee KY, Xiao B, Wang H, Yu F, Shen H-M, Liou Y-C. Death-associated protein 3 regulates mitochondrial-encoded protein synthesis and mitochondrial dynamics. Journal of Biological Chemistry. 2015; 290:24961-24974.

27. Sanchez A, de Vivo A, Tonzi P, Kim J, Huang TT, Kee Y. Transcription-replication conflicts as a source of common fragile site instability caused by BMI1-RNF2 deficiency. PLoS Genetics. 2020; 16:e1008524. 
28. Zhang L, Huo Q, Ge C, Zhao F, Zhou Q, Chen X, Tian H, Chen T, Xie H, Cui Y. ZNF143-mediated H3K9 trimethylation upregulates CDC6 by activating MDIG in hepatocellular carcinoma. Cancer Research. 2020;

29. Vaseva AV, Blake DR, Gilbert TS, Ng S, Hostetter G, Azam SH, Ozkan-Dagliyan I, Gautam P, Bryant KL, Pearce KH. KRAS suppression-induced degradation of MYC is antagonized by a MEK5-ERK5 compensatory mechanism. Cancer Cell. 2018; 34:807-822. e807.

30. Coppé J-P, Mori M, Pan B, Yau C, Wolf DM, Ruiz-Saenz A, Brunen D, Prahallad A, Cornelissen-Steijger $\mathrm{P}$, Kemper K. Mapping phospho-catalytic dependencies of therapy-resistant tumours reveals actionable vulnerabilities. Nature cell biology. 2019; 21:778-790.

31. Berger MD, Stintzing S, Heinemann V, Yang D, Cao S, Sunakawa Y, Ning Y, Matsusaka S, Okazaki S, Miyamoto Y. Impact of genetic variations in the MAPK signaling pathway on outcome in metastatic colorectal cancer patients treated with first-line FOLFIRI and bevacizumab: data from FIRE-3 and TRIBE trials. Annals of oncology. 2017; 28:2780-2785.

32. Cheng T-YD, Ambrosone CB, Hong C-C, Lunetta KL, Liu S, Hu Q, Yao S, Sucheston-Campbell L, Bandera EV, Ruiz-Narváez EA. Genetic variants in the mTOR pathway and breast cancer risk in African American women. Carcinogenesis. 2016; 37:49-55.

33. Cao H, Xiao C, Lu H, Yu H, Hong H, Guo C, Yuan J. MiR-129 reduces CDDP resistance in gastric cancer cells by inhibiting MAPK3. European review for medical and pharmacological sciences. 2019; 23:6478-6485.

34. Chi F, Wang Z, Li Y, Chang N. Knockdown of GINS2 inhibits proliferation and promotes apoptosis through the p53/GADD45A pathway in non-small-cell lung cancer. Bioscience Reports. 2020; 40:

35. Long J, Wang A, Bai Y, Lin J, Yang X, Wang D, Yang X, Jiang Y, Zhao H. Development and validation of a TP53-associated immune prognostic model for hepatocellular carcinoma. EBioMedicine. 2019; 42:363-374.

36. Fang SS, Guo JC, Zhang JH, Liu JN, Hong S, Yu B, Gao Y, Hu SP, Liu HZ, Sun L. A P53-related microRNA model for predicting the prognosis of hepatocellular carcinoma patients. Journal of Cellular Physiology. 2020; 235:3569-3578.

37. Zhang Z, Hong Y, Xiang D, Zhu P, Wu E, Li W, Mosenson J, Wu W-S. MicroRNA-302/367 cluster governs hESC self-renewal by dually regulating cell cycle and apoptosis pathways. Stem cell reports. $2015 ; 4: 645-657$.

38. Xu J, Lin H, Li G, Sun Y, Chen J, Shi L, Cai X, Chang C. The miR-367-3p increases sorafenib chemotherapy efficacy to suppress hepatocellular carcinoma metastasis through altering the androgen receptor signals. EBioMedicine. 2016; 12:55-67.

39. Cai H, Liu X, Zheng J, Xue Y, Ma J, Li Z, Xi Z, Bao M, Liu Y. Long non-coding RNA taurine upregulated 1 enhances tumor-induced angiogenesis through inhibiting microRNA-299 in human glioblastoma. Oncogene. 2017; 36:318-331.

40. Ye J, Zhang W, Liu S, Liu Y, Liu K. miR-363 inhibits the growth, migration and invasion of hepatocellular carcinoma cells by regulating E2F3. Oncology reports. 2017; 38:3677-3684. 
41. Chen GS, Zhou N, Li J-Q, Li T, Zhang Z-Q, Si Z-Z. Restoration of miR-20a expression suppresses cell proliferation, migration, and invasion in HepG2 cells. OncoTargets and therapy. 2016; 9:3067.

42. Zhang J, Zhu Y, Hu L, Yan F, Chen J. miR-494 induces EndMT and promotes the development of HCC (Hepatocellular Carcinoma) by targeting SIRT3/TGF- $/$ SMAD signaling pathway. Scientific reports. 2019; 9:1-13.

43. Jin F, Wang Y, Li M, Zhu Y, Liang H, Wang C, Wang F, Zhang C-Y, Zen K, Li L. MiR-26 enhances chemosensitivity and promotes apoptosis of hepatocellular carcinoma cells through inhibiting autophagy. Cell death \& disease. 2018; 8:e2540-e2540.

44. Zhang S, Ge W, Zou G, Yu L, Zhu Y, Li Q, Zhang Y, Wang Z, Xu T. MiR-382 targets GOLM1 to inhibit metastasis of hepatocellular carcinoma and its down-regulation predicts a poor survival. American journal of cancer research. 2018; 8:120.

45. Li Z-B, Chu H-T, Jia M, Li L. Long noncoding RNA LINC01139 promotes the progression of hepatocellular carcinoma by upregulating MYBL2 via competitively binding to miR-30 family. Biochemical and Biophysical Research Communications. 2020;

46. Aran D, Sirota M, Butte AJ. Systematic pan-cancer analysis of tumour purity. Nature communications. 2015; 6:8971.

47. Bindea G, Mlecnik B, Tosolini M, Kirilovsky A, Waldner M, Obenauf AC, Angell H, Fredriksen T, Lafontaine L, Berger A. Spatiotemporal dynamics of intratumoral immune cells reveal the immune landscape in human cancer. Immunity. 2013; 39:782-795.

48. Liu X, Wu S, Yang Y, Zhao M, Zhu G, Hou Z. The prognostic landscape of tumor-infiltrating immune cell and immunomodulators in lung cancer. Biomedicine \& Pharmacotherapy. 2017; 95:55-61.

49. Griffith JW, Sokol CL, Luster AD. Chemokines and chemokine receptors: positioning cells for host defense and immunity. Annual review of immunology. 2014; 32:659-702.

50. Borch TH, Donia M, Andersen MH, Svane IM. Reorienting the immune system in the treatment of cancer by using anti-PD-1 and anti-PD-L1 antibodies. Drug discovery today. 2015; 20:1127-1134.

51. Rotte A, Jin J, Lemaire V. Mechanistic overview of immune checkpoints to support the rational design of their combinations in cancer immunotherapy. Annals of Oncology. 2018; 29:71-83.

52. Granier C, De Guillebon E, Blanc C, Roussel H, Badoual C, Colin E, Saldmann A, Gey A, Oudard S, Tartour E. Mechanisms of action and rationale for the use of checkpoint inhibitors in cancer. ESMO open. $2017 ;$ 2:e000213.

\section{Figures}


A

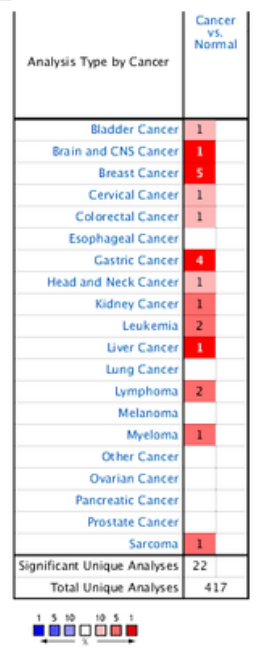

B

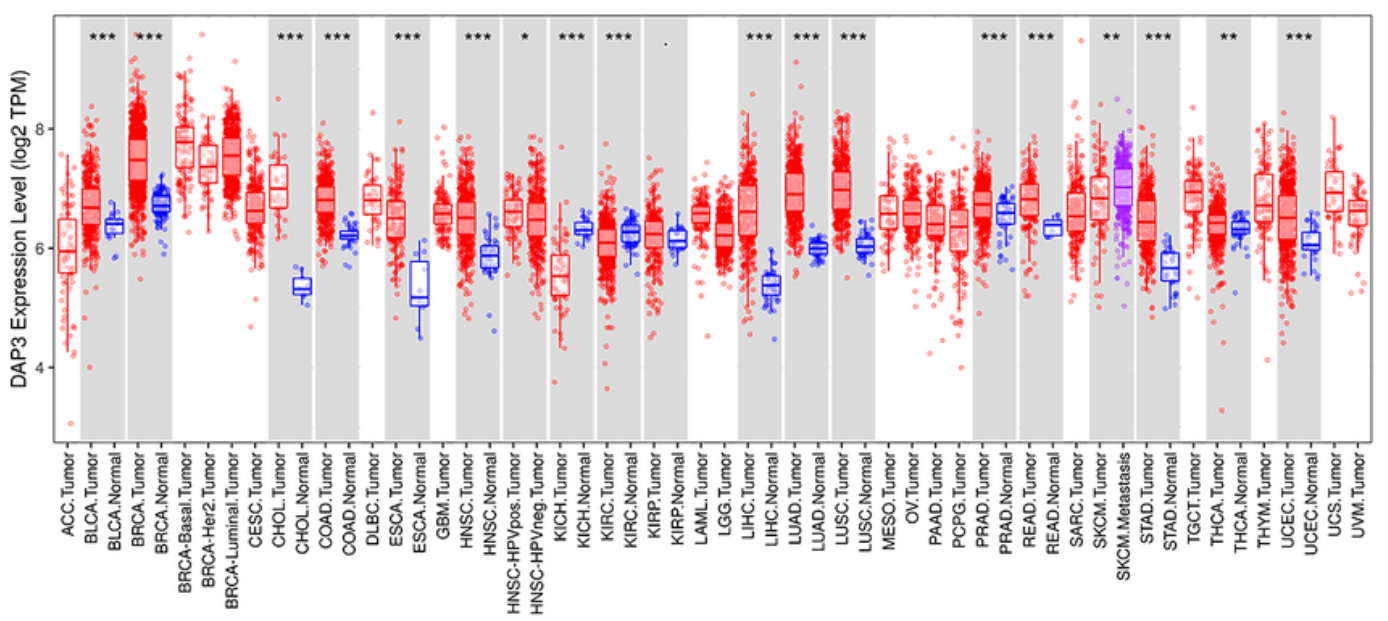

\section{Figure 1}

The expression of DAP3 in various human cancers. (A) The low or high mRNA expression of DAP3 in various human cancer tissues was compared to that in normal tissues using Oncomine database. (B) The DAP3 expression was detected from TCGA database via TIMER. Note: ${ }^{*} P<0.05,{ }^{\star *} \mathrm{P}<0.01,{ }^{\star \star *} \mathrm{P}<$ 0.001 . 

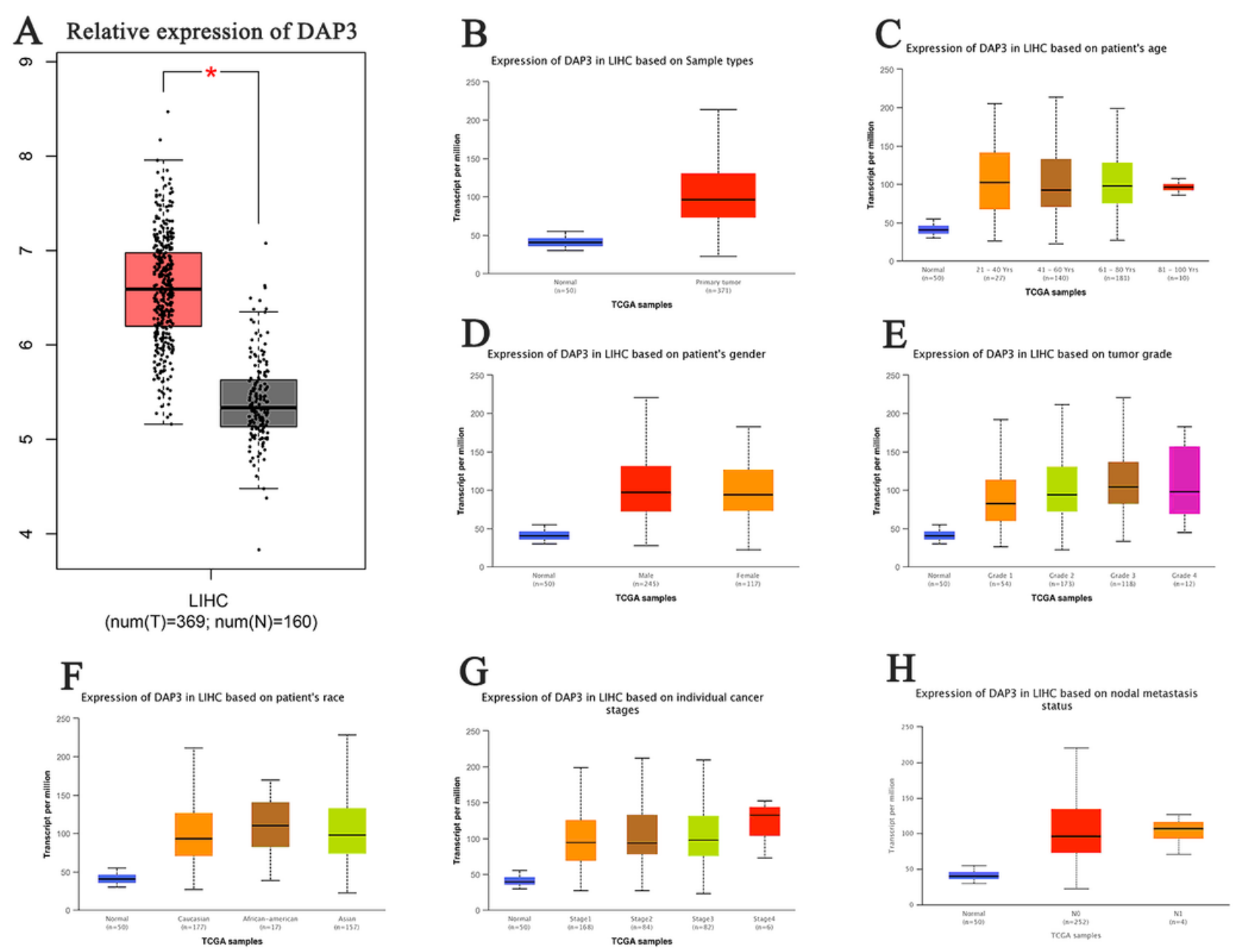

\section{Figure 2}

DAP3 expression in hepatocellular carcinoma (HCC). (A) The mRNA level of DAP3 was significantly higher in HCC tissues than that in normal tissues using GEPIA. (B) The DAP3 transcription level was remarkably increased in liver cancer tissues compared with that in liver normal tissues by UALCAN. (C) Boxplot showing relative expression of DAP3 in normal individuals of any ethnicity or in LIHC patients of Caucasian, African-American or Asian ethnicity. (D) Boxplot showing relative expression of DAP3 in normal individuals of either gender or male or female LIHC patients. (E) Boxplot showing relative expression of DAP3 in normal individuals of any age or in LIHC patients aged $21-40,41-60,61-80$, or 81-100 yr. (F) Boxplot showing relative expression of DAP3 in normal individuals or in LIHC patients in stages 1, 2, 3 or 4. (G) Boxplot showing relative expression of DAP3 in normal individuals or LIHC patients with grade 1, 2, 3 or 4 tumors. (H) Boxplot showing relative expression of DAP3 in normal individuals or in LIHC patients in with or without nodal metastasis. Figure $(\mathrm{C}-\mathrm{H})$ were conducted using UALCAN. Note: * $\mathrm{P}$ $<0.05, \star \star \mathrm{P}<0.01, \star \star \star \mathrm{P}<0.001$. 
A

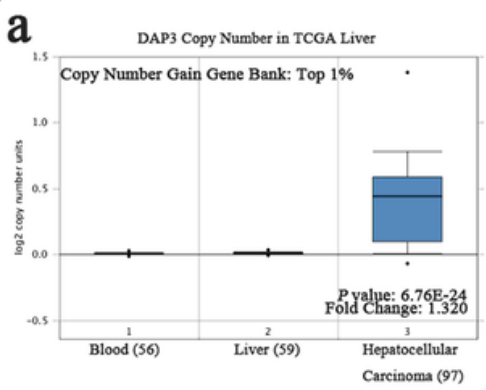

B

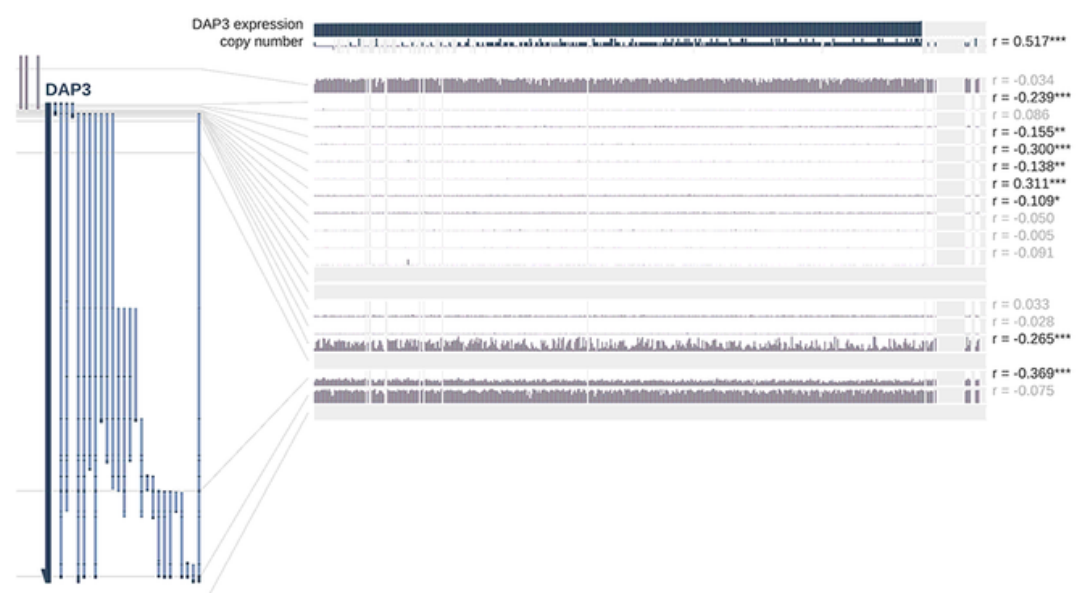

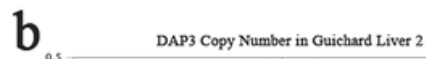

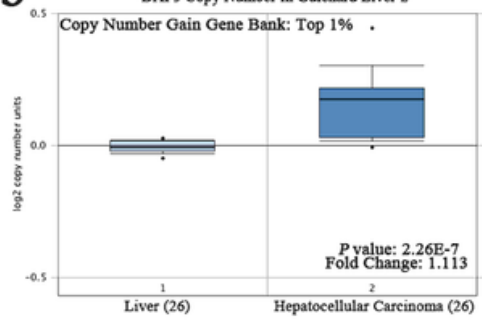

C DAP3 Copy Number in Guichard Liver

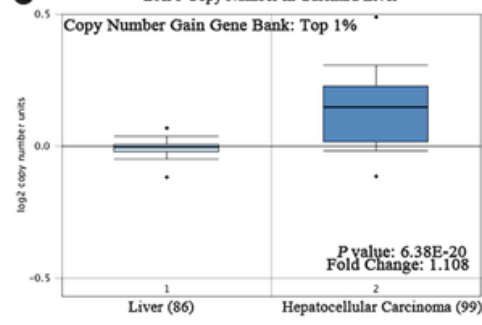

$\mathrm{C}$

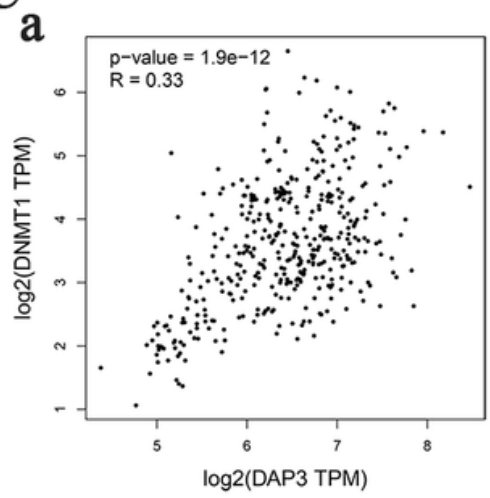

b

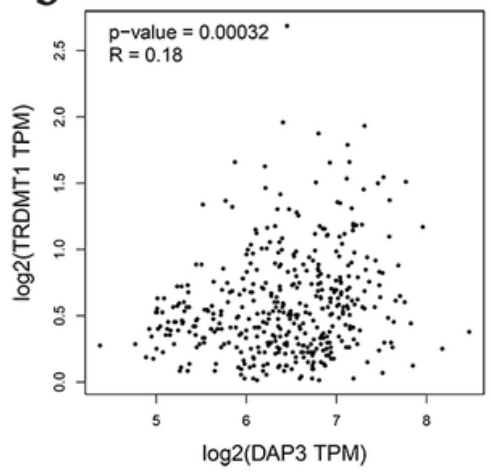

c

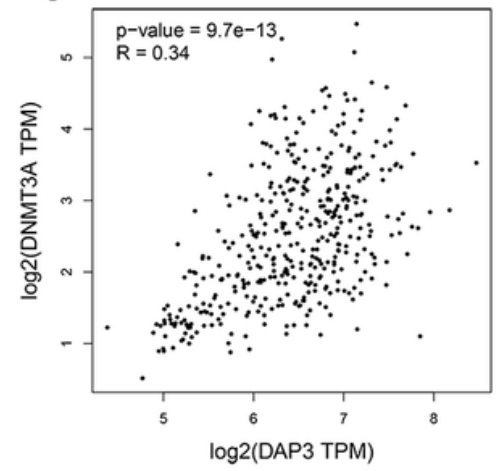

d

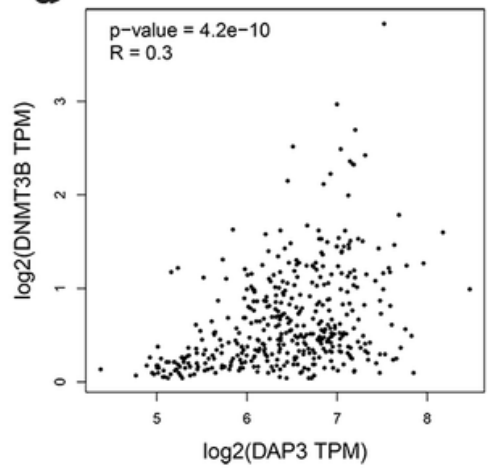

\section{Figure 3}

The epigenetic modifications of TASP1 in HCC. (A) Box plot showing DAP3 copy number in The Cancer Genome Atlas (TCGA) Liver and Guichard Liver datasets, respectively. (B) The methylation level of DAP3 promoter regions was modified using MEXPRESS. (C) The DAP3 expression was significantly correlated with DNA methyltransferase (DNMT) expression via GEPIA. 

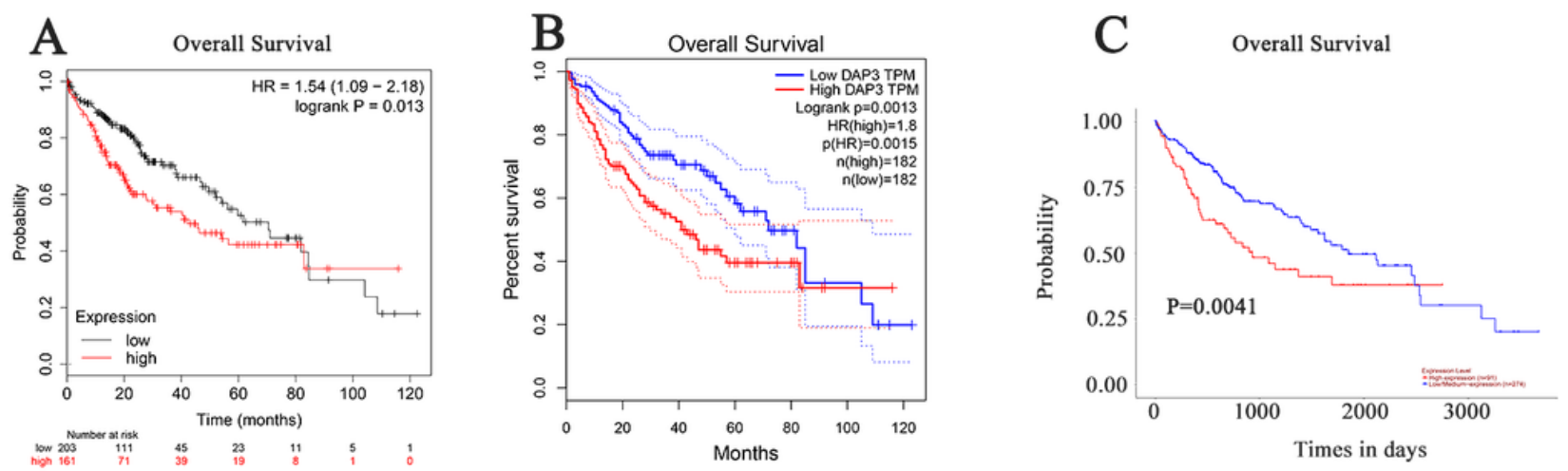

\section{Figure 4}

The DAP3 expression as a prognostic biomarker in HCC. (A) High DAP3 expression was correlated with poor overall survival (OS) in HCC patients using Kaplan-Meier Plotter database. (B) High DAP3 expression was correlated with poor OS in HCC patients using GEPIA. (C) High DAP3 expression was correlated with poor OS in HCC patients using UALCAN. 


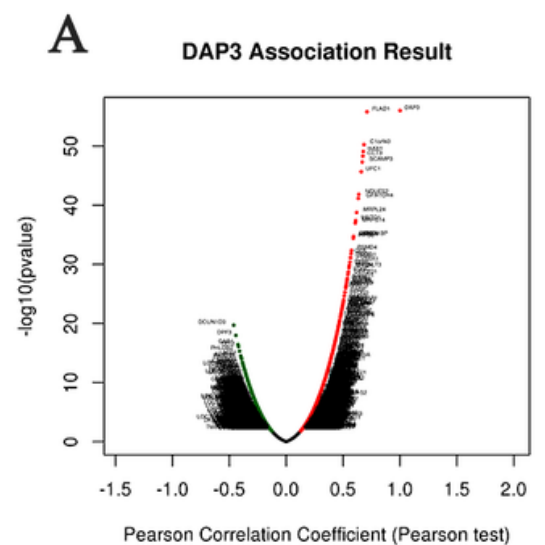

B

\section{C}
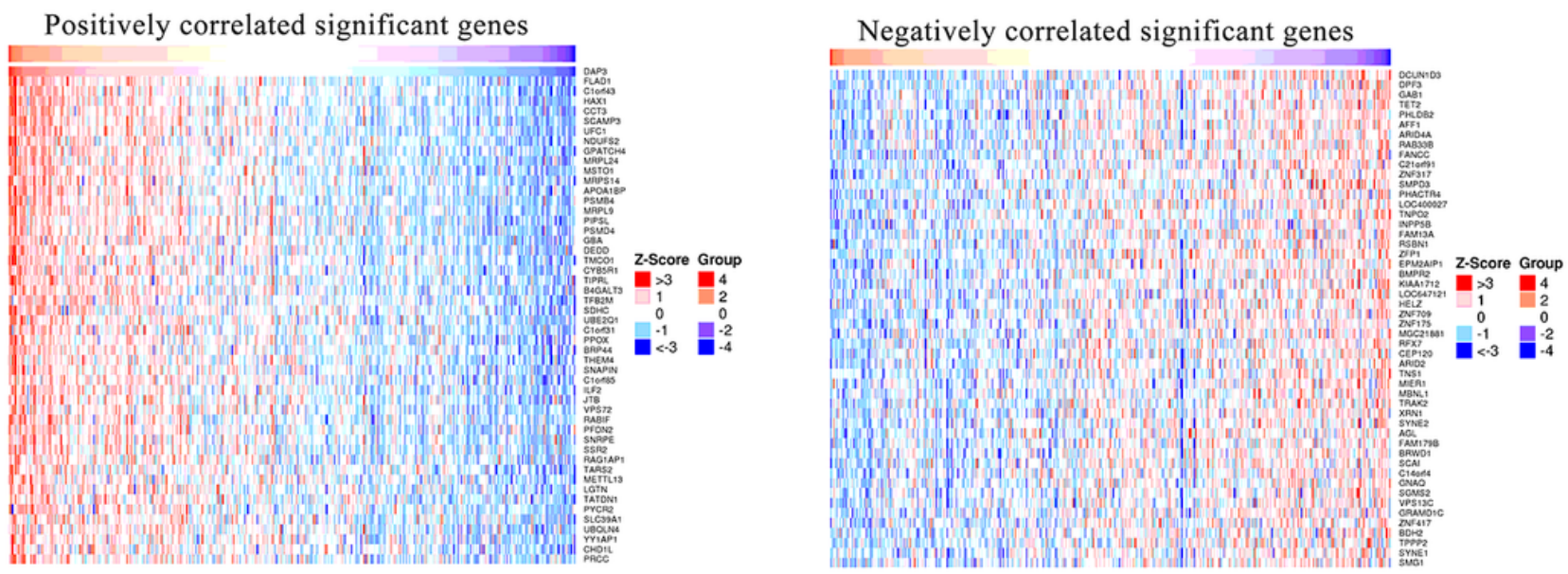

\section{Figure 5}

Genes differentially expressed in correlation with DAP3 in HCC (LinkedOmics). (A) The volcano plot was used to show the correlations between DAP3 and genes differentially expressed in LIHC by Pearson test. (B-C) Heat maps showing top 50 genes positively or negatively correlated with DAP3 in LIHC 

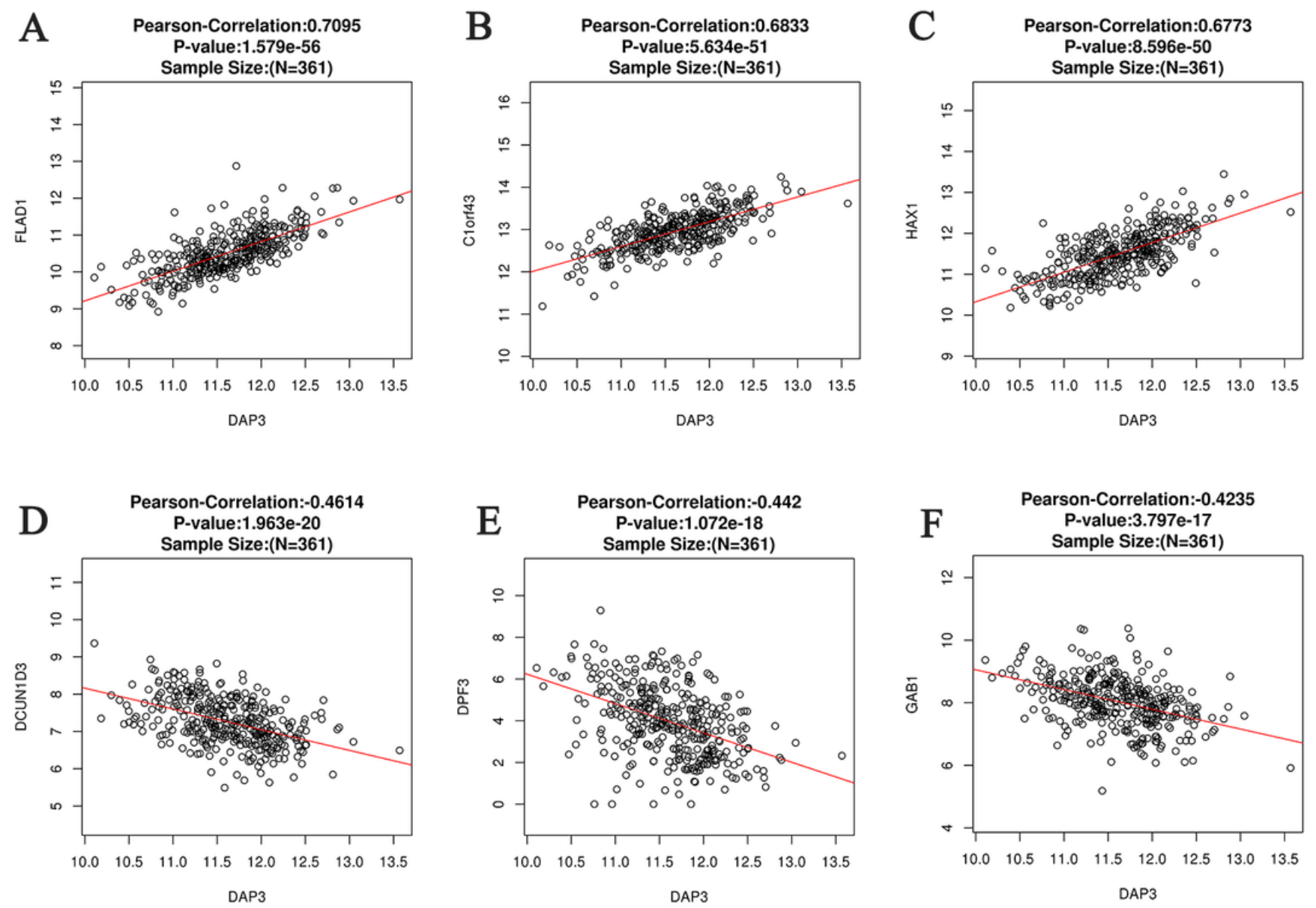

\section{Figure 6}

Verification of the correlation between DAP3 expression and the top 3 differentially expressed genes via GEPIA. (A-C) DAP3 expression was positively correlated with FLAD1, C1 orf43, and HAX1 (top 3 genes). (DF) DAP3 expression was negatively correlated with DCUN1D3, DPF3, and GAB1 (top 3 genes). 

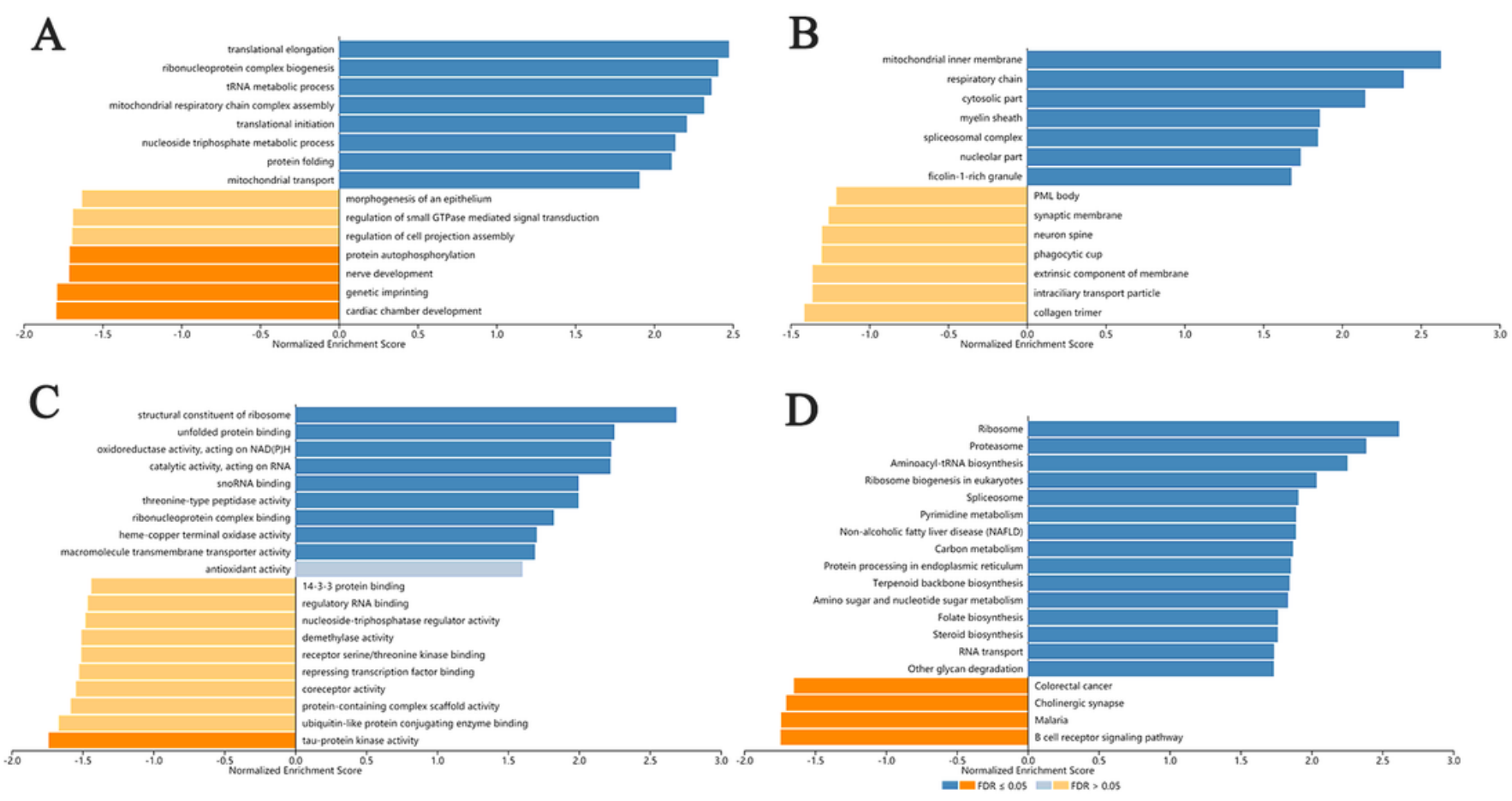

\section{Figure 7}

Biological function of DAP3 correlated genes in HCC using LinkedOmics. GSEA was used to perform the significantly enriched GO annotations and KEGG pathways of DAP3 correlated genes in HCC. (A) Cellular components. (B) Biological processes. (C) Molecular functions. (D) KEGG pathway analysis.

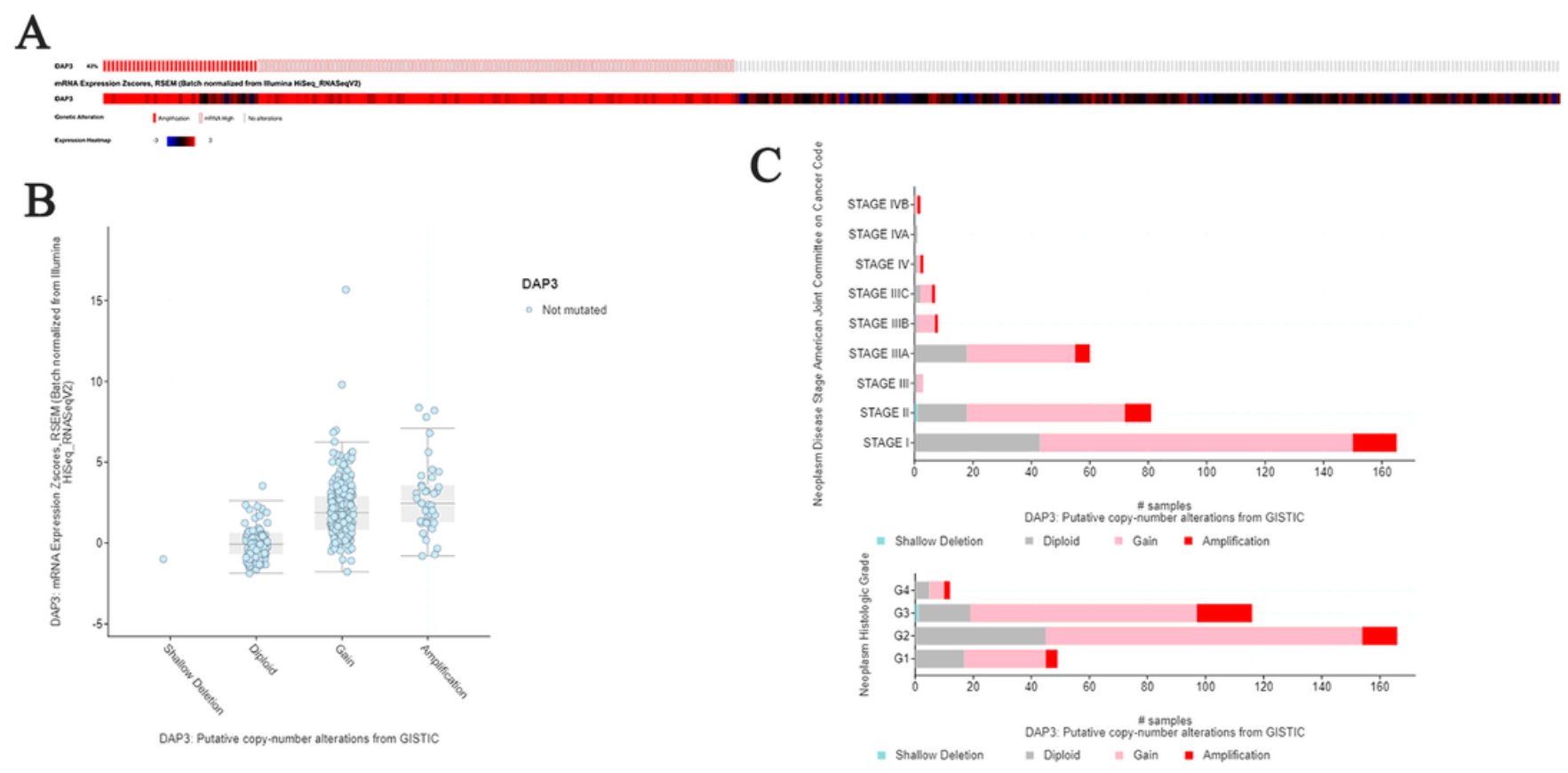


DAP3 genomic alterations in HCC (cBioPortal). (A) OncoPrint of DAP3 alterations in LIHC cohort. The different types of genetic alterations are highlighted in different colors. (B) DAP3 expression in different DAP3 CNV groups. DAP3 amplification (AMP) group has a significantly higher expression level. (C) Distribution of DAP3 CNV frequency in different stage and grade subgroups. The percentage number on the right of the bar indicates the ratio of patients with DAP3 gain or AMP in all this subgroup patients.
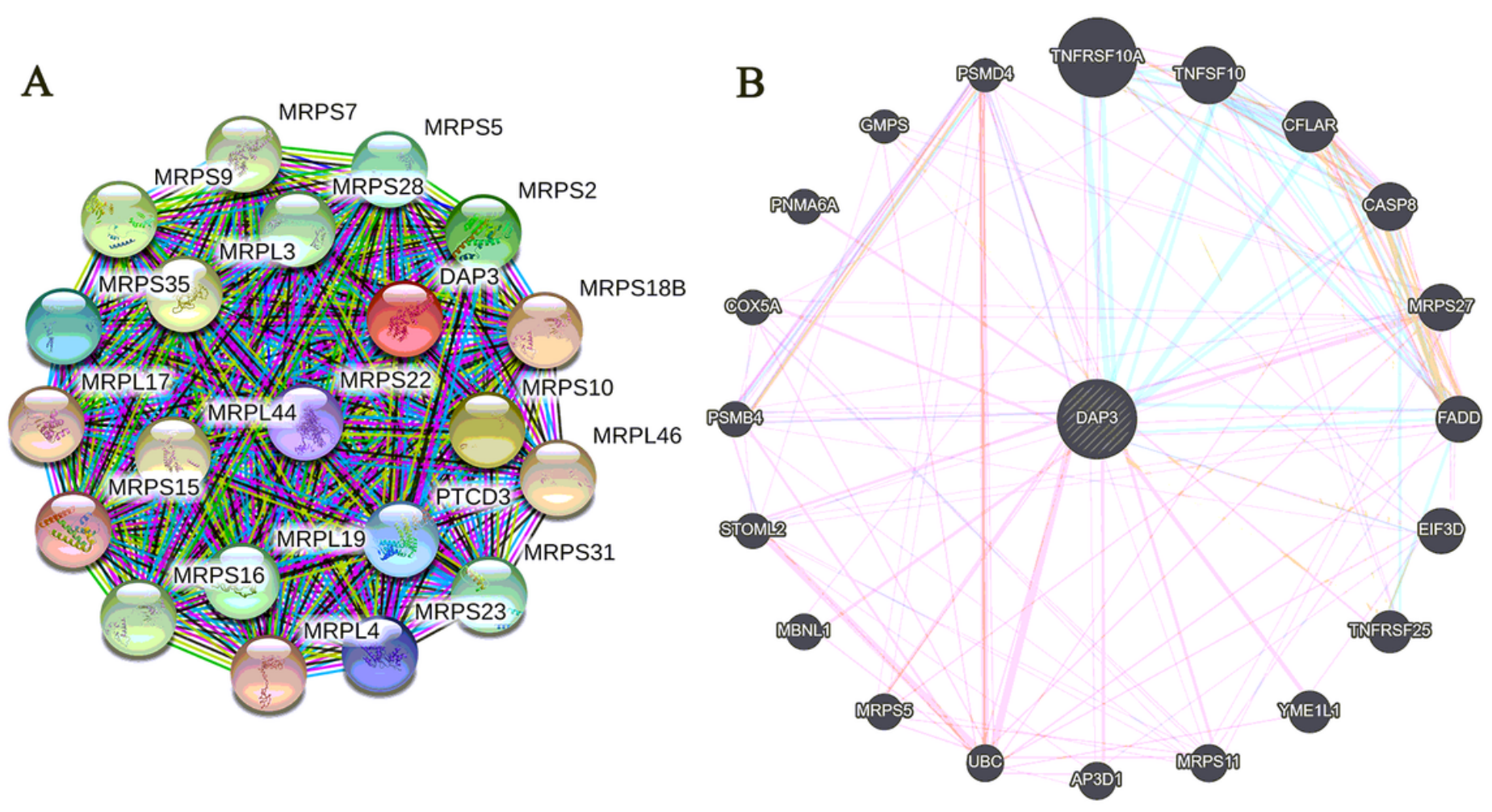

\section{Figure 9}

Protein-protein interaction network of DAP3 networks. (A) On line tool of STRING database analysis of the PPI network for the differentially expressed genes correlated with DAP3. (B) Co-expression network of DAP3 was shown using GeneMANIA. 

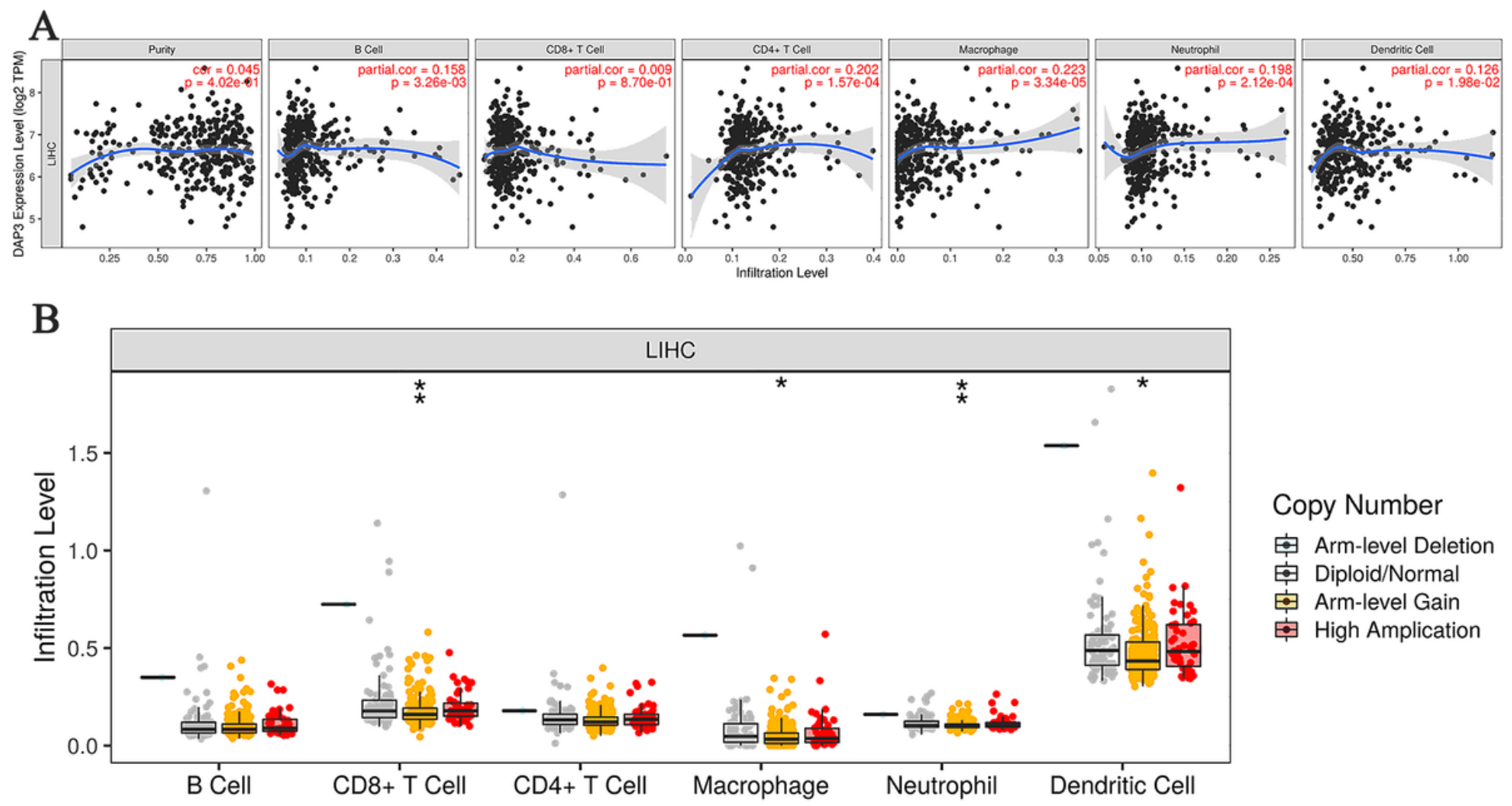

Copy Number

审 Arm-level Deletion

审 Diploid/Normal

Arm-level Gain

审 High Amplication

\section{Figure 10}

Correlations of DAP3 expression with immune infiltration level in HCC using TIMER. (A) The expression of DAP3 in LIHC has no significant correlations with tumor purity and CD8囚T cells, and DAP3 expression is positively related to the infiltrating levels of immune cells (B cells, CD4囚T cells, macrophages, neutrophils, and dendritic cells). (B) DAP3 CNV affects the infiltrating levels of CD8+T cells, macrophages,

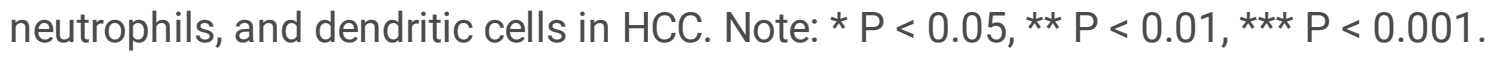



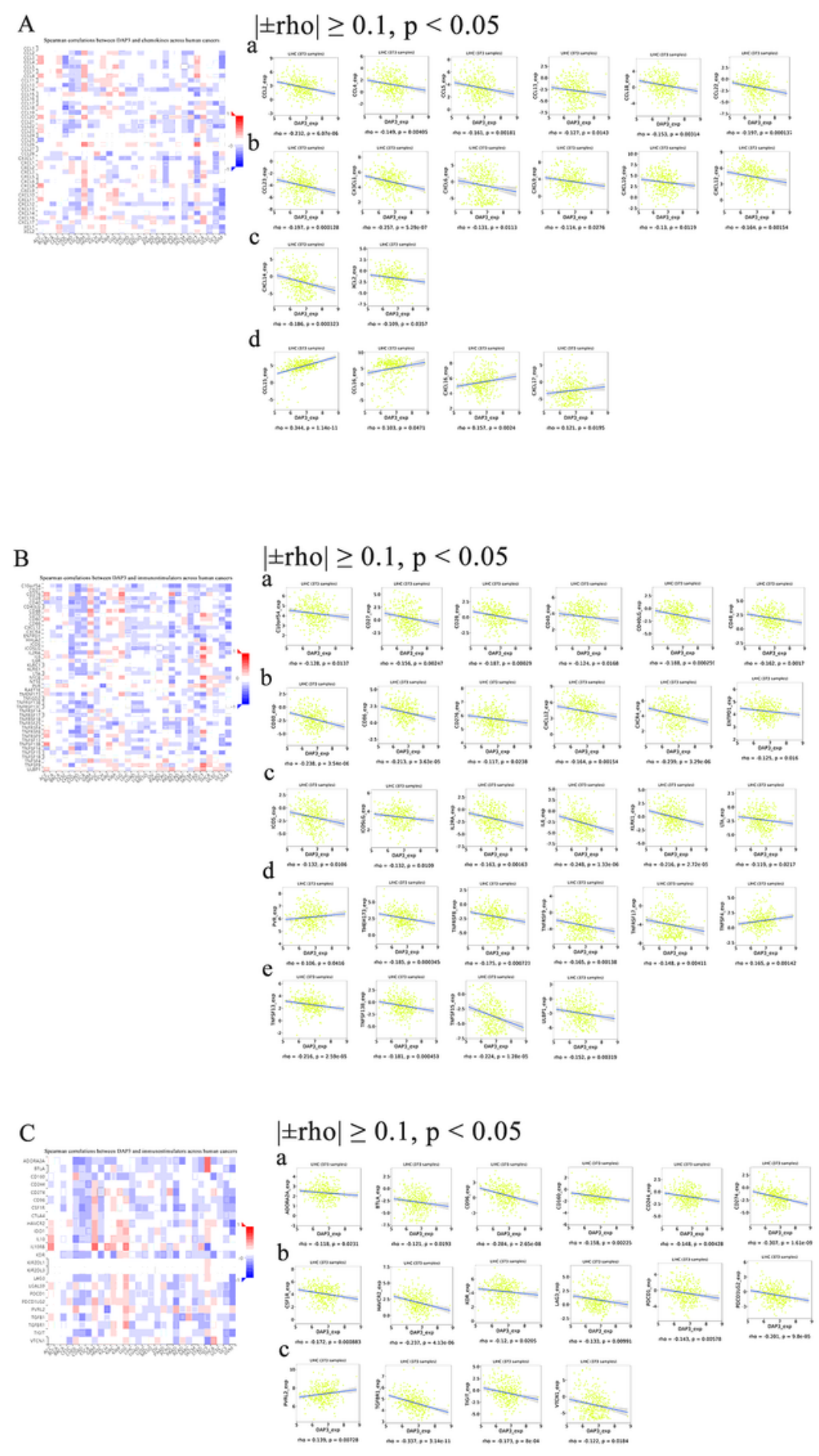

\section{Figure 11}

Correlations between DAP3 expression and three cancer-related immune factor types in TISIDB. (A) Correlation between DAP3 and chemokines in HCC. (B) Correlation between DAP3 and immunoinhibitors in HCC. (C) Correlation between DAP3 and immunostimulators in HCC.

\section{Supplementary Files}


This is a list of supplementary files associated with this preprint. Click to download.

- DAP3supplement.docx

- supplementarytable1.xlsx

- supplementarytable2.xlsx

- supplementarytable3.xlsx

- supplementarytable4.xlsx

- supplementarytable5.xlsx

- supplementarytable6.xlsx

- supplementarytable7.xlsx

- supplementarytable8.xlsx 